\title{
Clinical Analysis of Apatinib in the Treatment of Patients with Residual Tumor after Radical Chemoradiotherapy for Locally Advanced Cervical Cancer
}

\author{
Jun Jiang ${ }^{*}$, Wei Hong Wei and Tao Xu
}

Department of Radiation Oncology, the First People's Hospital of Foshan, Foshan 528000, China

\begin{abstract}
There is no standard treatment for locally advanced cervical cancer, patients with residual tumor after radical concurrent chemoradiotherapy. This study was to investigate the short-term efficacy and safety of the targeted drug apatinib mesylate in patients with tumor residual after radical chemoradiotherapy for locally advanced cervical cancer. Eight patients with residual tumors after localized concurrent chemoradiotherapy with locally advanced cervical cancer were treated with apatinib (250 mg once daily, orally). The short-term efficacy and safety of the eight patients treated with apatinib were initially evaluated. Total Effectiveness (ORR) $37.5 \%$ and disease control rate (DCR) $100.0 \%$. The toxicity and side effects were light, mainly manifested as $37.5 \%$ of hand-foot syndrome, $37.5 \%$ of proteinuria, $25.0 \%$ of hypertension, $12.5 \%$ of fatigue, $12.5 \%$ of rash, and $12.5 \%$ of vomiting. No serious toxic side effects associated with the drug were observed. Apatinib mesylate can be safely used in patients with residual tumor after radical chemoradiotherapy for locally advanced cervical cancer. The short-term effect is positive and the side effects are low.
\end{abstract}

Keywords: Apatinib, tyrosine kinase inhibitor, cervical cancer, radiation therapy, chemotherapy, outcome.

\section{INTRODUCTION}

Cervical cancer is one of the most common malignant tumors in women and ranks second among female malignant tumor mortality [1]. According to statistics, in 2012, there were about 527,600 new cases and 265,700 deaths, which seriously threatened women's health [2]. The NCCN guidelines recommend that the recommended treatment for patients with locally advanced cervical cancer who are inoperable is radical concurrent chemoradiotherapy. Patients with residual tumors after radical concurrent chemoradiotherapy are in good condition and can tolerate further treatment, but there are currently no standard treatment options for these patients. Tumor residue causes great psychological stress on patients, which seriously affects the quality of life of patients. There is an urgent need for a safe and effective treatment to improve their quality of life and extend their survival.

In recent years, molecular targeted drugs for the purpose of inhibiting the survival of blood vessels have been widely studied and applied with good curative effect but minor toxicities. Studies have shown that Apatinib (YN968D1, Aitan), a novel oral small molecule VEGFR-2 tyrosine kinase inhibitor [3-10], produces therapeutic effects in a variety of solid tumors while bearing high safety and good tolerance. At present, the application of apatinib in the treatment of cervical cancer has not been reported.

*Address correspondence to this author at the Department of Radiation Oncology, the First People's Hospital of Foshan, Foshan 528000, China;

Tel: 18038865018; E-mail: jjun@fsyyy.com

ISSN: 1929-2260 / E-ISSN: 1929-2279/20
This study retrospectively analyzed the clinical data of 8 patients with residual tumor after radical chemoradiotherapy for locally advanced cervical cancer treated with apatinib, and explored patients with residual tumor after radical chemoradiotherapy for locally advanced cervical cancer. The clinical value of the disease provides a basis for the development of individualized treatment plans for patients with residual tumors after radical chemoradiotherapy for locally advanced cervical cancer.

\section{INFORMATION AND METHODS}

\subsection{Case Selection and General Information}

Data of eight patients with residual tumors after radical chemoradiotherapy for locally advanced cervical cancer who were treated with apatinib in First People's Hospital of Foshan (Foshan, China) from December 01, 2017 to September 30, 2018, was chosen for this study. The presence of residual tumor mass was confirmed on the basis of MRI examination carried out 1 month after the end of radiotherapy and chemotherapy, showing residual tumors with short diameter greater than or equal to $1 \mathrm{~cm}$. The ages of the patients included in the study ranged from 26 to 65 years old, with a median age of 49 years. The pathological types included 7 cases of squamous cell carcinoma and 1 case of adenocarcinoma. The 2009 FIGO staging criteria were used for staging of the cancer with 3 cases in stage IIB and 5 cases in stage III.

\subsection{Treatment Plan}

Apatinib mesylate tablets (Jiangsu Hengrui Pharmaceutical Co., Ltd., National Pharmaceutical 
Standard H20140103) were used to treat the patients in this study in a dose of $250 \mathrm{mg}$, once per day, half an hour after meal for one month. 1 cycle was comprised of treatment for 1 month. Patients were given a total of 1-3 cycles.

\subsection{Evaluation of Efficacy}

The efficacy was evaluated 1 month after the start of the drug administration with the effects evaluation once every 3 months according to the new WHO objective response evaluation criteria (Response Evaluation Criteria in Solid Tumors, RECIST) [11], mentioned below:

Complete remission (CR): The lesion completely subsided for at least 4 weeks, and no new lesions appeared;

Partial remission (PR): The sum of the longest diameters of the lesions is reduced by at least $30 \%$, maintained for at least 4 weeks and no new lesions appear;

Progression of disease (PD): at least a $20 \%$ increase in the sum of the longest diameters of the lesion or the appearance of new lesions;

Stable disease (SD): The lesions did not significantly shrink on the basis of PR, and there was no significant increase on the basis of $P D$, which was between the two.

Recent efficacy evaluations include: objective rate of effectiveness (ORR) and disease control rate (DCR), where ORR $=(C R+P R) /$ total number of cases $x$
$100 \%, D C R=(C R+P R+S D) /$ total number of cases $\times 100 \%$.

\subsection{Toxicity Side Reaction Evaluation Criteria}

Toxic side effects of the treatment with Apatinib were evaluated according to the National Cancer Institute-Common Toxicity Criteria (NCl-CTC 3.0) standard.

\section{RESULTS}

\subsection{Short-Term Efficacy}

Out of total 8 patients, efficacy was found to be CR for 1 case, PR for 2 cases, SD for 5 cases, PD for 0 case, ORR $37.5 \%$, DCR $100.0 \%$ (Table 1). During the follow-up period of median 15 mouths all 8 patients survived and no tumor progression was observed.

Table 1 The short-term efficacy of 8 patients with residual tumor after radical chemoradiotherapy for locally advanced cervical cancer.

\subsection{Adverse Reactions}

Adverse reactions mainly include: hand-foot syndrome, proteinuria, hypertension, fatigue, rash, vomiting, etc. The incidence of hand-foot syndrome was $37.5 \%$ ( $37.5 \%$ for grades 1 to 2,0 for grades 3 to 4), $37.5 \%$ for proteinuria ( $37.5 \%$ for grades 1 to 2,0 for grades 3 to 4$)$, hypertension. The incidence rate was $25.0 \%$ (15.0\% for grade 1 and 0 for grade 2 to 4$)$, the incidence of fatigue was $12.5 \%$ (12.5\% for grade 1 and 0 for grade 2 to 4 ), and the incidence of rash was $12.5 \%(1.5 \%$ was $12.5 \%)$. The grades of 2 to 4 are 0$)$,

Table 1: Short Effectiveness

\begin{tabular}{|c|c|c|c|c|c|c|}
\hline Therapeutic effect & CR & PR & SD & PD & ORR & DCR \\
\hline Number of cases & 1 & 2 & 5 & 0 & 3 & 8 \\
\hline Percentage (\%) & 12.5 & 25.0 & 62.5 & 0 & 37.5 & 100.0 \\
\hline
\end{tabular}

Table 2: The Major Toxicities [n(\%)]

\begin{tabular}{|c|c|c|c|c|c|}
\hline Toxic side reaction & $\mathbf{I}$ & II & III & IV & total \\
\hline Hand and Foot Syndrome & $2(25.0)$ & $1(12.5)$ & $0(0)$ & $0(0)$ & $3(37.5)$ \\
\hline Proteinuria & $2(25.0)$ & $1(12.5)$ & $0(0)$ & $0(0)$ & $3(37.5)$ \\
\hline High blood pressure & $2(25.0)$ & $0(0)$ & $0(0)$ & $0(0)$ & $2(25.0)$ \\
\hline Weakness & $1(12.5)$ & $0(0)$ & $0(0)$ & $0(0)$ & $1(12.5)$ \\
\hline Rash & $1(12.5)$ & $0(0)$ & $0(0)$ & $0(0)$ & $1(12.5)$ \\
\hline Vomiting & $1(12.5)$ & $0(0)$ & $0(0)$ & $0(0)$ & $1(12.5)$ \\
\hline
\end{tabular}


and the incidence of vomiting is $12.5 \%$ (1.5\% is $12.5 \%$, and 2 to 4 is 0 ). No treatment for class III/IV toxicity was found in the treatment group, and there was no treatment-related death see Table 2.

Table 2 Major adverse reactions in 8 patients with residual tumor after radical chemoradiotherapy for locally advanced cervical cancer [example (\%)].

\section{DISCUSSION}

Local advanced cervical cancer, radical synchronous radiotherapy and chemotherapy after tumor residual patients, there is no standard treatment. Castelnau-marchand $\mathrm{P}$ et al. [12] reviewed and analyzed 9 patients with concurrent radiotherapy and chemotherapy for local advanced cervical cancer. Results although the 4-year tumor-free survival rate (DFS) was improved, the incidence of delayed complications was significantly increased. Dan sni et al. [13] Meta analysis of the efficacy of surgical treatment for patients with locally advanced cervical cancer after chemoradiotherapy and chemotherapy. The results showed that surgical treatment of patients with locally advanced cervical cancer after radiotherapy and chemotherapy failed to improve the overall survival rate, and surgical treatment of patients with locally advanced cervical cancer after radiotherapy and chemotherapy was not recommended.

Cai Maohuai [14] used high-intensity ultrasound focused thermocoagulation (HIFU) combined with systemic chemotherapy to treat 17 patients with local residual or recent recurrence after radiotherapy for cervical cancer, and observed tumor volume changes 3 months after treatment. The results showed that 2 cases of CR, 9 cases PR, 4 cases SD, and ORR was $88.2 \%$. The recent high efficiency, but the long-term efficacy has not been reported further.

Kim HS et al. [15] analyzed 37 patients with locally advanced cervical cancer undergoing radical chemotherapy after radical chemoradiotherapy, including 3 cycles of concurrent chemotherapy and 3 cycles of consolidation chemotherapy. The chemotherapy regimen was paclitaxel (135 mg/m (2)), carboplatin (AUC 5.0). Studies have shown that patients have lower rates of CR, DFS, and OS compared to previous reports. Dueñas-González A et al. [16] in a phase III clinical study of patients with locally advanced cervical cancer, analyzed the efficacy and side effects of consolidation chemotherapy versus single-agent concurrent chemoradiotherapy after concurrent chemoradiotherapy. The study showed that the former was superior to the latter in 3-year progression-free survival (PFS), overall survival (OS), and disease progression time (TTP), but the former had a significant increase in grade 3 and 4 toxicity, including 2 causes. Patients with toxic side effects died. Seiji Mabuchi et al. [17] retrospectively analyzed the clinical data of 82 patients with stage IIIB/IVA cervical cancer who underwent concurrent chemotherapy and single-agent concurrent chemoradiotherapy with dualagent concurrent chemoradiotherapy. For example, 52 patients with single-agent concurrent chemoradiotherapy were treated with paclitaxel and carboplatin once a week. The chemotherapy regimen was paclitaxel, carboplatin, one cycle every 3-4 weeks, a total of 3 cycles; the latter synchronous chemotherapy regimen is cisplatin, once a week. The analysis pointed out that the former improved the local control rate and survival rate.

Tumor formation, development and metastasis are inseparable from the formation of new blood vessels, so inhibition of neovascularization is the key to tumor treatment. Since the VEGF/VEGFR-2 signaling pathway plays an important role in tumor angiogenesis, blocking this signaling pathway and inhibiting tumor angiogenesis has become a new hot spot for tumor targeted therapy [18]. Apatinib is a small molecule antiangiogenic agent, a novel small molecule tyrosine kinase inhibitor that selectively inhibits the tyrosine kinase of VEGFR-2 [18]. By competitively binding VEGFR-2, blocking VEGF binding to VEGFR-2, inhibiting VEGFR-2 phosphorylation, down-regulating the phosphorylation level of its downstream extracellular signal-regulated kinase, and then inhibiting tumor angiogenesis for therapeutic purposes.

Approved by the State Food and Drug Administration on December 13, 2014, apatinib was used to treat advanced gastric cancer or gastricesophageal junction adenocarcinoma. A number of clinical studies have demonstrated [3-10] that apatinib has a clear effect on a variety of solid tumors, with high safety and good tolerance. The level of VEGFR-2 expression in tumor tissues can be used as a sensitivity to the treatment of apatinib. One of the indicators. Ji et al. [23] reported that an elderly male patient with malignant fibrous histiocytoma (MFH) with high expression of VEGFR-2 mRNA was treated with apatinib $500 \mathrm{mg} / \mathrm{d}$ for 2 months. Mitigation (PR).

Most patients with cervical cancer who have residual tumor after radical chemoradiotherapy are not sensitive to radiotherapy, and the tumor tissue is 
hypoxic, which creates conditions for the application of the anti-angiogenic drug apatinib. In addition, the patient's physical condition is generally good, and there is a strong desire for further treatment. Therefore, we used apatinib for the first time in patients with cervical cancer who had residual tumor after radical concurrent chemoradiotherapy for efficacy evaluation and safety observation.

The results of this study showed that the total effective rate of patients was $37.5 \%$, the disease control rate was $100.0 \%$, and the toxicity was mild. The main toxic side effects are hand-foot syndrome, proteinuria, hypertension, fatigue, rash, vomiting and so on. The incidence of proteinuria was $37.5 \%$, all of which were grade I-II, which was relieved after active treatment. Hypertension, fatigue, rash, vomiting, etc. occurred in grade 1 with an incidence of $25.0 \%, 12.5 \%$, $12.5 \%$, and $12.5 \%$, respectively. No II-IV response was found, which was relieved after symptomatic treatment. No treatment-related deaths. No hematological toxicity and bleeding occurred.

\section{CONCLUSION}

Patients with cervical cancer who have residual tumors after radical concurrent chemoradiotherapy with apatinib have a short-term efficacy, low toxicity and high safety. The clinical significance of this treatment plan needs to be further confirmed by accumulating more and higher-level clinical trials.

\section{REFERENCES}

[1] Parkhurst JO, Vulimiri M. Cervical cancer and the global health agenda: insights from multiple policy-analysis frameworks. Glob public Health 2013; 8(10): 1093-108. https://doi.org/10.1080/17441692.2013.850524

[2] Torre LA, Bray F, Siegel RL, Ferlay J, Lortet-Tieulent J, Jemal A. Global cancer statistics, 2012. CA Cancer J Clin 2015; 65(2): 87-108 https://doi.org/10.3322/caac.21262

[3] Li J, Zhao X, Chen L, et al. Safety and pharmacokinetics of novel selective vascular endothelial growth factor receptor-2 inhibitor YN968D1 in patients with advanced malignancies [J]. BMC cancer 2010; 10: 529. https://doi.org/10.1186/1471-2407-10-529

Li J, Qin S, Xu J, et al. Apatinib for chemotherapy-refractory advanced metastatic gastric cancer: results from a randomized, placebo-controlled, parallel-arm, phase II trial [J]. Journal of clinical oncology: official journal of the American Society of Clinical Oncology 2013; 31(26): 321925.

https://doi.org/10.1200/JCO.2013.48.8585

[5] Li J, Qin S, Xu J, et al. Randomized, double-blind, placebocontrolled phase III trial of apatinib in patients with chemotherapy-refractory advanced or metastatic adenocarcinoma of the stomach or gastro-esophageal junction[J]. J Clin Oncol 2016; (3): 35-38.
Qin SK. Phase III study of apatinib in advanced gastric cancer: A randomized, double-blind, placebo-controlled trial. [J]. Journal Of Clinical Oncology 2014; 32(15). https://doi.org/10.1200/jco.2014.32.15 suppl.4003

$\mathrm{Hu} \mathrm{X}$, Cao J, Hu W, et al. Multicenter phase II study of apatinib in non-triple-negative metastatic breast cancer [J]. BMC cancer 2014; 14: 820.

https://doi.org/10.1186/1471-2407-14-820

[8] $\mathrm{Hu} \mathrm{X}$, Zhang J, Xu B, et al. Multicenter phase II study of apatinib, a novel VEGFR inhibitor in heavily pretreated patients with metastatic triple-negative breast cancer [J]. International journal of cancer 2014; 135(8): 1961-9. https://doi.org/10.1002/ijc.28829

[9] Li Z, Kai L, Meizhen S, et al. Randomized, double-blind placebo-controlled, multicenter phase II clinical trial of apatinib mesylate in advanced non-squamous non-small cell lung cancer [M]. The 15th National Clinical Proceedings of the Oncology Conference and the 2012 CSCO Academic Annual Conference. Beijing 2012; 24.

[10] Qin SK. Apatinib in Chinese patients with advanced hepatocellular carcinoma: A phasell randomized, open-label trial[J]. J Clin Oncol 2014; 32(15): 17-20. https://doi.org/10.1200/jco.2014.32.15 suppl.4019

[11] Kozloff M, Yood MU, Berlin J, Flynn PJ,Kabbinavar FF Purdie DM, Ashby MA, Dong W, Sugrue MM, Grothey A. Clinical outcomes associated with bevacizumab-containing treatment of metastatic colorectal cancer: the BRiTE observational cohort study. Oncologist 2009; 14: 862-870. https://doi.org/10.1634/theoncologist.2009-0071

[12] Castelnau-Marchand P, Chargari C, Bouaita R, et al. What to expect from immediate salvage hysterectomy following concomitant chemoradiation and image-guided adaptive brachytherapy in locally advanced cervical cancer[J]. Cancer Radiother 2015; 19(8): 710-7. https://doi.org/10.1016/j.canrad.2015.05.025

[13] Shi D, Liang Z, Zhang C, et al. The effect of surgery on the survival status of patients with locally advanced cervical cancer after radiotherapy/chemoradiotherapy: a metaanalysis[J]. BMC Cancer 2018; 18: 308.

https://doi.org/10.1186/s12885-018-4232-x

[14] Maohuai C, Hongyu G, Jun X, et al. Short-term efficacy of HIFU combined with systemic chemotherapy in the treatment of 17 cases of cervical cancer after radiotherapy[J]. Shanghai Medical Imaging 2007; 16(4): 303-304.

[15] Kim HS, Kim MK, Kim HJ, et al. Phase IIStudy of Consolidation Chemotherapy after Adjuvant or Primary Concurrent Chemoradiation Using Paclitaxel and Carboplatin to Treat High-Risk Early-Stage or Locally Advanced Cervical Cancer[J]. Epub 2012; 44(2): 97-103. https://doi.org/10.4143/crt.2012.44.2.97

[16] Dueñas-González A, ZarbáJJ, Patel F, et al. Phase III,openlabel, randomized study comparing concurrent gemcitabine plus cisplatin and radiation followed by adjuvant gemcitabine and cisplatin versus concurrent cisplatin and radiation in patients with stage IIB to IVA carcinoma of the cervix [J]. Epub 2011; 29(13): 1678-85. https://doi.org/10.1200/JCO.2009.25.9663

[17] Mabuchi S, Isohashi F, Okazawa $\mathrm{M}$, et al Chemoradiotherapy followed by consolidation chemotherapy involving paclitaxel and carboplatin and in FIGO stage IIIB/IVA cervical cancer patients[J]. Gynecol Oncol 2017; 28(1): e15.

https://doi.org/10.3802/jgo.2017.28.e15

[18] Hamerlik P, Lathia JD, Rasmussen R, et al. Autocrine VEGFVEGFR2-Neuropilin-1 signaling promotes glioma stem-like cell viability and tumor growth [J]. The Journal of experimental medicine 2012; 209(3): 507-20. https://doi.org/10.1084/jem.20111424 
[19] Tian S, Quan H T, Xie C Y, et al. YN968D1 is a novel and selective inhibitor of vascular endothelial growth factor receptor-2 tyrosine kinase with potent activity in vitro and in vivo [J]. Cancer Sci 2011; 102(7): 1374-80.

https://doi.org/10.1111/j.1349-7006.2011.01939.x

[20] Song S, Ewald AJ, Stallcup W, et al. PDGFRbeta+ perivascular progenitor cells in tumours regulate pericyte differentiation and vascular survival [J]. Nature cell biology 2005; 7(9): 870-9.

https://doi.org/10.1038/ncb1288

[21] Irby RB, Yeatman TJ. Role of Src expression and activation in human cancer [J]. Oncogene 2000; 19(49): 5636-42. https://doi.org/10.1038/sj.onc.1203912
[22] Heinrich MC, Blanke CD, Druker BJ, et al. Inhibition of KIT tyrosine kinase activity: a novel molecular approach to the treatment of KIT-positive malignancies [J]. Journal of clinical oncology: official journal of the American Society of Clinical Oncology 2002; 20(6): 1692-703. https://doi.org/10.1200/JCO.2002.20.6.1692

[23] Ji G, Hong L, Yang P. Successful treatment of advanced malignant fibrous histiocytoma of the right forearm with apatinib: a case report [J]. Onco Targets Ther 2016; 9(3): 643-647.

https://doi.org/10.2147/OTT.S96133

() 2020 Jiang et al.; Licensee Neoplasia Research.

This is an open access article licensed under the terms of the Creative Commons Attribution Non-Commercial License (http://creativecommons.org/licenses/by-nc/3.0/) which permits unrestricted, non-commercial use, distribution and reproduction in any medium, provided the work is properly cited. 